\title{
Simulations of volumetric hydrogen storage capacities of nanoporous carbons: Effect of dispersion interactions as a function of pressure, temperature and pore width
}

\author{
I. Cabria \\ Departamento de Física Teórica, Atómica y Óptica, Universidad de Valladolid, 47011, Valladolid, Spain
}

\section{A R T I C L E I N F O}

Article history:

Received 28 November 2018

Received in revised form

6 March 2019

Accepted 8 March 2019

Available online $\mathrm{xxx}$

Keywords:

Hydrogen storage

Hydrogen physisorption

Nanoporous carbons

Dispersion interactions

Graphene

DFT

\begin{abstract}
A B S T R A C T
Simulations of the hydrogen storage capacities of activated carbons require an accurate treatment of the interaction of a hydrogen molecule physisorbed on the graphitic-like surfaces of nanoporous carbons, which is dominated by the dispersion interactions. These interactions are described accurately by high level quantum chemistry methods such as the Coupled cluster method with single and double excitations and a non-iterative correction for triple excitations $(\operatorname{CCSD}(\mathrm{T}))$, but those methods are computationally very expensive for large systems and massive simulations. Density functional theory (DFT) based methods that include dispersion interactions are less accurate, but computationally less expensive. Calculations of the volumetric hydrogen storage capacities of nanoporous carbons, simulated as benzene and graphene slit-shaped pores, have been carried out, using a quantum-thermodynamic model of the physisorption of $\mathrm{H}_{2}$ on surfaces and the interaction potential energy curves of $\mathrm{H}_{2}$ physisorbed on benzene and graphene obtained using the $\operatorname{CCSD}(\mathrm{T})$ and second order Møller-Plesset (MP2) methods and the 14 most popular DFT-based methods that include the dispersion interactions at different levels of complexity. The effect of the dispersion interactions on the DFT-based volumetric capacities as a function of the pressure, temperature and pore width is evaluated. The error of the volumetric capacities obtained with the quantum-thermodynamic model and each method is also calculated and analyzed.
\end{abstract}

@ 2019 Hydrogen Energy Publications LLC. Published by Elsevier Ltd. All rights reserved.

\section{Introduction}

An alternative fuel to the fossil fuels used in the motor vehicles is hydrogen. This fuel can be stored in tanks either as compressed gas at high pressures or liquefied under cryogenic temperatures. These types of storage are expensive and have drawbacks. Many research efforts are devoted to a third type of storage: on solid materials. Storage of hydrogen on solid materials can be achieved via chemisorption or physisorption. Light metal hydrides store hydrogen through the chemisorption process. These materials have high hydrogen storage capacities, but slow sorption kinetics and high decomposition. Different methods have been studied to overcome these

E-mail address: ivan.cabria@uva.es. 
disadvantages [1-9]. The storage through the physisorption mechanism has been widely investigated on porous solid materials, such as metal-organic frameworks (MOFs) [10-13], covalent organic frameworks (COFs) [14-17], organic polymers [18-21] and nanoporous carbons [22-26]. A promising group of porous materials are the nanoporous carbons. These materials have low densities, high porosities, high specific surface areas and nanometer size pores. This group includes activated carbons, carbon nanofibers, carbide-derived carbons, schwarzites, carbon nanofoams, carbon nanohorns, carbon nano-onions, carbon nanotubes, etc.

Theoretical simulations of the hydrogen storage capacities of nanoporous carbons have been performed to understand the experimental results and to predict/design new carbon-based materials [27-46]. These simulations require an accurate description of the interaction of a $\mathrm{H}_{2}$ molecule with the graphitic-like surfaces of these materials. The hydrogen storage capacity of nanoporous carbons depends mainly on the interactions around the binding region of the interaction potential energy curve $V(z)$, where $z$ is the molecule-surface distance, but also on the tail region, the region far from the carbon surface [39-42]. The interaction between a hydrogen molecule and a surface is dominated by the dispersion forces, which are accurately described by high level quantum chemistry methods such as the $\operatorname{CCSD}(\mathrm{T})$ method [47]. However, those methods are computationally very expensive and not practical for large systems and massive calculations. DFTbased methods that include the dispersion interactions at different levels of complexity are less accurate, but they are computationally less expensive and more practical. The use of those DFT-based methods, instead of the high level quantum chemistry methods, implies to reach a balance between accuracy and computer time and resources.

There is a lack of comprehensive comparisons in the scientific literature of the hydrogen storage capacities of nanoporous carbons obtained with DFT functionals and high level quantum chemistry methods. The studies involved only a few DFT functionals or high level methods [31,39-46], but they were not systematic and did not study the inclusion of the dispersion interactions at different levels of complexity. A more comprehensive comparison of the hydrogen storage capacities obtained with the DFT-based methods that include the dispersion interactions, especially the recent non-local functionals, and those obtained with high level methods, such as $\operatorname{CCSD}(\mathrm{T})$, is necessary. The present research is a comparison of the 14 most popular DFT-based methods that include dispersion interactions to assess their performance to simulate accurately the volumetric storage capacities of nanoporous carbons and the effect of including the dispersion interactions. The MP2 method and three DFT-based methods that do not include the dispersion interactions are also included in the comparison.

Experimental results of the hydrogen storage capacities, the hydrogen adsorption energies, etcof nanoporous carbons vary greatly, because the pores and surfaces of each material are very different. The theoretical results also vary greatly, depending on the model used to simulate the nanoporous carbon, and on the theoretical method. Hence, to clarify matters, two very specific models of nanoporous carbons have been chosen: the benzene and graphene slit pores. Calculations of the volumetric hydrogen storage capacities of nanoporous carbons, simulated as benzene and graphene slit pores, have been carried out in the present research, using a quantum model of the thermodynamic equilibrium of the two phases of hydrogen molecules inside pores [41-45], and the interaction potential energy curves $\mathrm{V}(\mathrm{z})$ of $\mathrm{H}_{2}$ on two different graphitic-like surfaces, benzene and graphene, obtained with the DFT-based methods and the $\operatorname{CCSD}(\mathrm{T})$ and MP2 methods. It should be remarked that the $\operatorname{CCSD}(\mathrm{T}), \mathrm{MP} 2$ and DFT storage capacities are calculated by this quantum model in an indirect way, through the interaction potential energy curves $V(z)$ obtained with these methods.

The quantum-thermodynamic, QT, model has been applied to different types of carbon-based nanopores [41-45] and uses the interaction potential energy between a hydrogen molecule and the surface of the pore. Except the interaction potential energy curves of $\mathrm{H}_{2}$ on graphene obtained with VWN and PW91, the rest of the interaction potential energy curves were published in a previous paper [29]. $\operatorname{CCSD}(\mathrm{T})$ and MP2 were used to calculate $\mathrm{V}(\mathrm{z})$ only on benzene. The calculations of $\mathrm{V}(\mathrm{z})$ that were done with basis sets, included the correction of the basis set superposition error, BSSE. The calculations of $\mathrm{H}_{2}$ on graphene performed with the VWN and PW91 functionals were done in the same conditions as the calculations performed and published with another functionals [29].

To evaluate the effects of the dispersion interactions on the volumetric capacities as a function of the pressure, temperature and pore width, the theoretical volumetric capacities obtained with the QT model and with the MP2 and 17 DFT-based methods are compared with the capacities obtained with the high precision $\operatorname{CCSD}(\mathrm{T})$ method, in the case of benzene slit pores, and with experiments in the case of graphene slit pores. A quantitative estimation of the error of the theoretical volumetric capacities obtained with the QT model has been also carried out. Previous investigations of the storage capacities obtained with $\operatorname{CCSD}(\mathrm{T}), \mathrm{MP} 2$ and some DFT-based methods did not include an estimation of the error of the calculated capacities [31,39-45]. These comparisons and estimations of errors also allows us to find out which DFT-based methods yield the most accurate volumetric capacities.

The sections of this paper are organized as follows. In section 2 the QT model is explained, together with the geometry of the slit pores. Section 3 consists on explaining the calculation of the errors of the QT model. Section 4 is devoted to the selected reference volumetric capacities, used to calculate the errors. Section 5 consists on the comparison and discussion of the volumetric capacities of benzene and graphene slit pores obtained by means of the QT model and using 17 DFT-based methods, and the $\operatorname{CCSD}(\mathrm{T})$ and MP2 methods. The errors of the theoretical volumetric capacities are discussed in Section 6. The conclusions of the comparison are presented and summarized in the last section. 


\section{Methodology: the quantum-thermodynamic, QT,} model

\section{Phases of hydrogen inside a pore}

The basis of the quantum-thermodynamic, QT, model is the equation of the thermodynamic equilibrium between the adsorbed and compressed phases of hydrogen inside a pore. The compressed phase is also called bulk gas and free gas phase throughout the scientific literature. The amount of hydrogen adsorbed on the surface of the pore is determined by the thermodynamic equilibrium between the two phases, at a given temperature and external pressure.

Fig. 1 is a depiction of the two phases of hydrogen inside a pore. The adsorbed phase is the sum of the blue and purple regions and the compressed phase is the red region. The masses of the phases are not proportional to the areas of Fig. 1. The compressed phase is composed by molecules that do no interact with the surfaces of the pores and only interact with other molecules [48-50]. The adsorbed phase is composed by molecules physisorbed on the surface of the pores (the blue and purple regions in Fig. 1). The molecules of the purple mass are normally present in the compressed phase in the volume occupied by the adsorbed phase. The molecules of the blue mass are not present in the compressed phase and they are called the molecules of the excess mass.

The volumetric capacities of these phases and of the stored hydrogen are calculated using the QT model. The original model used the ideal gas equation of state, EOS [45], and the revised model uses the Mills-Younglove EOS [44]. Avdeenkov et al. have published recently a similar model that shows quite similar results to the revised model [31].

\section{Geometry of the pores: benzene and graphene slit pores}

The geometry of the pore is an important part of the QT model. Different shapes of pores: cylindrical, spherical and planar-parallel or slit, have been studied within the QT model [41-45].

Many regions of the nanoporous carbons are flat graphiticlike surfaces parallel to each other and separated by a few nanometers, as reported by experiments [51]. Those regions are called slit pores. Therefore, the pore geometry most used to simulate nanoporous carbons is the slit pore: two infinite

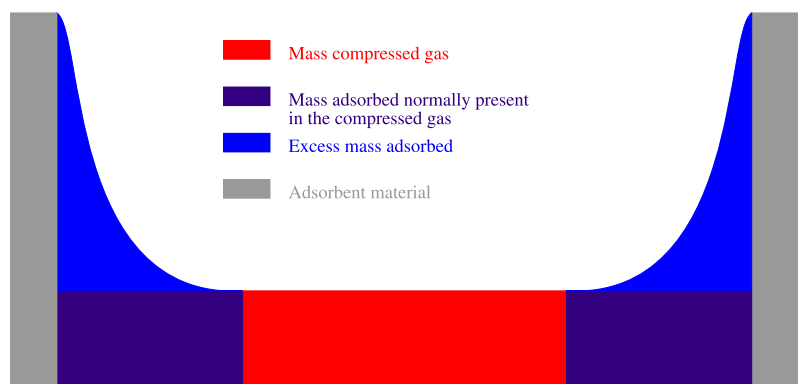

Fig. 1 - Depiction of the masses of the adsorbed and compressed phases of $\mathrm{H}_{2}$ gas inside a slit pore. The masses are not proportional to the region areas. parallel graphitic-like flat surfaces separated a certain distance $w$, called the pore width (See Fig. 2). Two different graphitic-like surfaces have been studied: benzene and graphene. The corresponding pores are called benzene and graphene slit pores, respectively.

\section{Equations and steps of the QT model}

There are several steps to calculate the volumetric capacity of the adsorbed phase within the QT model [41-45]. The first step is the calculation of the quantum states of $\mathrm{H}_{2}$ in the pore potential by solving the corresponding Schrödinger equation. There are two flat parallel layers in a slit pore, and therefore, the pore potential is the sum of the potentials of the two layers separated a distance $w: V(z)+V(w-z)$ (See Fig. 3), where $V(z)$ is the interaction potential energy between $\mathrm{H}_{2}$ and a single benzene or graphene layer, $\mathrm{z}$ is the $\mathrm{H}_{2}$-layer surface distance and $w$ is the distance between the layers, also called the pore width.

The second step consists on the calculation of the partition function of the adsorbed hydrogen phase, $z_{\text {ads }}$, at temperature $T$, using the energy eigenvalues $\varepsilon_{i}$ of the quantum states of the molecule in the pore. The partition function of the adsorbed phase is given by:

$Z_{\text {ads }}=\sum e^{-\beta \varepsilon_{i}}$

where $\beta=1 / k_{\mathrm{B}} \mathrm{T}$ and $k_{\mathrm{B}}$ is the Boltzmann constant. The equilibrium constant between the adsorbed and compressed phases, confined in the volume $\mathrm{V}$ of the pore, is given by

$K_{\text {eq }}=Z_{\text {ads }} / Z_{\text {com }}$,

where $Z_{\text {com }}$ is the partition function of the compressed, nonadsorbed phase. In the case of a slit pore of width $w$, this partition function is obtained as

$Z_{\text {com }}=\left(w-2 w_{\text {excl }}\right) \sqrt{2 \pi m k_{\mathrm{B}} T / h^{2}}$,

where $m$ is the mass of the hydrogen molecule and $w_{\text {excl }}$ is an exclusion distance due to the repulsive part of $V(z)$ near the pore layers. The exclusion distance is defined as the location where the repulsive part of $\mathrm{V}(\mathrm{z})$ is one $\mathrm{eV}$.
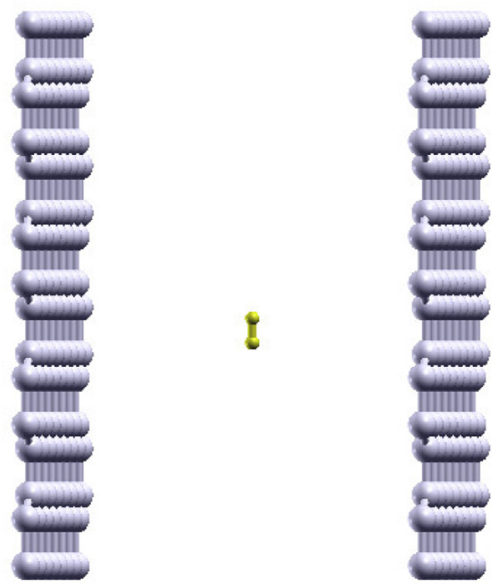

Fig. 2 - Graphene slit pore with one hydrogen molecule inside. The two graphene sheets are flat and parallel. 


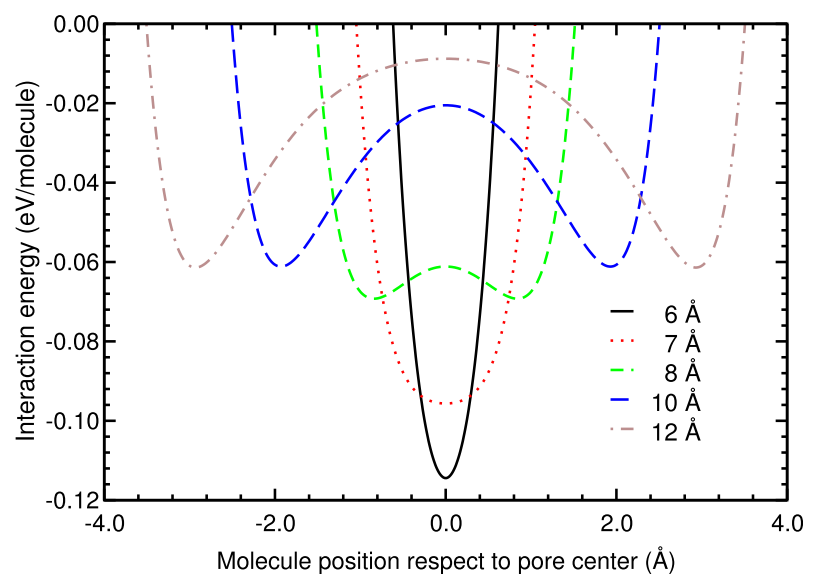

Fig. 3 - Interaction potential energy, $\mathbf{V}(\mathbf{z})+\mathbf{V}(\boldsymbol{w}-\mathbf{z})$, between a $\mathrm{H}_{2}$ molecule and graphene slit pores of different widths $w: 6,7,8,10$ and $12 \AA$, obtained in rev-vdW-DF2 calculations.

The equilibrium constant, $K_{\text {eq }}$, is related to the pressures of the compressed and adsorbed phases, $P_{\text {com }}$ and $P_{\text {ads }}$, respectively, and to the temperature $\mathrm{T}$ by means of the equation:

$\ln K_{\text {eq }}=\frac{1}{R T} \int_{P_{\text {com }}}^{P_{\text {ads }}} v_{\text {mol }}(P, T) d P$.

The pressure of the compressed phase, $P_{\text {com }}$, is the pressure $P$ exerted externally to fill the adsorbent material with hydrogen gas. It is also sometimes called the external pressure. The molar volume $v_{\mathrm{mol}}(P, T)$ in Eq. (4) is given by the EOS of hydrogen. The EOS used in the QT model is the empirical Mills-Younglove EOS, explained in Refs. [41-44].

The third step consists on solving Eq. (4) and obtaining the pressure of the adsorbed phase, $P_{\text {ads }}$, for each value of $P_{\text {com }}=P$ and $\mathrm{T}$. Then, the molar volume in $\mathrm{L} / \mathrm{mol}$ of the adsorbed hydrogen phase, $v_{\text {mol }}\left(P_{\text {ads }}, T\right)$, is obtained using $P_{a d s}$ and the EOS of hydrogen.

Finally, the volumetric capacity of the adsorbed phase in $\mathrm{kg}$ of hydrogen/L is given by:

$v_{c}=\frac{a}{v_{\text {mol }}\left(P_{\text {ads }}, T\right)} \frac{V_{\text {adsorbed }}}{V_{\text {pore }}}$,

where $a$ is a constant to convert from moles to $\mathrm{kg}$ of hydrogen, $V_{\text {adsorbed }}$ is the volume of the adsorbed phase and $V_{\text {pore }}$ is the pore volume.

These are the definitions and units of the volumetric capacities in this paper. Throughout this paper only the volumetric capacity of the adsorbed phase is calculated and analyzed, because the effects of the pore potential occur in the adsorbed phase, not in the compressed phase. The volumetric capacity $v_{c}$ in Eq. (5) depends on $P, T$ and $w$, through Eqs. (1)-(4).

The QT model establishes a relationship between the storage capacities and the pore potential energy. In Fig. 3 the rev-vdW-DF2 potential energies of pores of five different widths are plotted. The pore potential shows a single deep minimum for narrow slit pores ( 6 and $7 \AA$ ), and two separated minima for larger pore widths (8, 10 and $12 \AA)$, which correspond to the two separated flat graphene sheets. This relationship is another form to indicate that the QT model calculates indirectly the storage capacities, through a given or input interaction potential energy curve $\mathrm{V}(\mathrm{z})$, and not directly.

\section{Interaction potential energy curves of benzene and graphene slit pores}

The hydrogen storage volumetric capacities of benzene slit pores have been calculated with the QT model and using the interaction potential energy curves $\mathrm{V}(\mathrm{z})$ of $\mathrm{H}_{2}$ on benzene obtained with the methods $\operatorname{CCSD}(\mathrm{T})$ [47] and MP2 [52], and with 17 DFT-based methods: PBE [53,54], VWN [55], PW91 [56], $\mathrm{PBE}+$ DCACP [57-60], B97D [61,62], PBE-D2 [61], PBE-TS [63], PBE-XDM [64,65], vdW-DF [66], optB88-vdW [67], optB86b-vdW [68], vdW-DF-cx [69], vdW-DF-C09 [70], vdW-DF2 [71], vdWDF2-C09 [72], rev-vdW-DF2 [73] and RVV10 [74]. The molecule was on top of the center of the benzene molecule and perpendicular to the benzene-surface. The interaction potential energy curves were calculated and published previously, with the BSSE (Basis Set Superposition Error) correction when using finite basis [29].

The volumetric capacities of graphene slit pores have been calculated using the interaction potential energy curves $\mathrm{V}(\mathrm{z})$ of $\mathrm{H}_{2}$ on graphene obtained with 16 DFT-based methods: PBE [53,54], VWN [55], PW91 [56], PBE + DCACP [57-60], PBE-D2 [61], PBE-TS [63], PBE-XDM [64,65], vdW-DF [66], optB88-vdW [67], optB86b-vdW [68], vdW-DF-cx [69], vdW-DF-C09 [70], vdW-DF2 [71], vdW-DF2-C09 [72], rev-vdW-DF2 [73] and RVV10 [74]. Except the VWN and PW91 energy curves, the rest of the curves have been published previously [29]. The $\mathrm{H}_{2}$ molecule was on top of the center of a graphene hexagon, parallel to the graphene surface and parallel to two $\mathrm{C}-\mathrm{C}$ bonds of the hexagon. This was the site and orientation of $\mathrm{H}_{2}$ on graphene with the lowest energy.

\section{Calculation of the error of the volumetric capacities obtained with the QT model}

The main source of error of the QT model is the interaction potential energy $V(z) \cdot \operatorname{CCSD}(T)$ calculations of $\mathrm{H}_{2}$ on benzene provide an accurate $V(z)$ potential and hence, the $\operatorname{CCSD}(\mathrm{T})$ volumetric capacities are considered the accurate and reference capacities in the case of benzene slit pores and will be used to compare and to calculate the error of the capacities obtained with other methods. The PBE-D2 volumetric capacities are the reference in the case of graphene slit pores, because of reasons that will be explained in a later section.

The theoretical volumetric capacities depend strongly on the pressure, temperature and pore width and their errors should also depend on those variables. Experiments usually report the volumetric capacities as a function of pressure and for a fixed temperature, the so-called volumetric isotherms. On another hand, the experimental volumetric capacities are not related to specific pore widths, because nanoporous carbons are not composed by pores of specific widths, but for pores of different widths and with different relative abundances, such as these carbons have a Pore Size Distribution, 
PSD. The theoretical volumetric capacities, however, are calculated for specific pore widths. The PSD of a nanoporous carbon is not always known and depends on the type of nanoporous carbon. These reasons lead to a practical definition of the errors of the volumetric capacities (or more exactly, the volumetric isotherms) as an average over pressures and pore widths.

The RMSE (T; M), the Root-Mean-Square Error, and RMSPE (T; M), the Root-Mean-Square Percentage Error, of the volumetric isotherm obtained with the method $M$ at temperature $T$ are defined, respectively, as the following averages over pressures and pore widths:

$\operatorname{RMSE}(T ; M)=\sqrt{\frac{1}{N L} \sum_{i=1}^{N} \sum_{j=1}^{L} \Delta(i, j, T)^{2}}$

$\operatorname{RMSPE}(\mathrm{T} ; \mathrm{M})=100 \sqrt{\sum_{i=1}^{N} \sum_{j=1}^{L} \frac{\Delta(i, j, T)^{2}}{\operatorname{NL}\left(v_{c}\left(P_{i}, T, w_{j} ; \operatorname{Ref}\right)\right)^{2}}}$,

where $\Delta(i, j, T)$ is given by:

$\Delta(i, j, T)=v_{c}\left(P_{i}, T, w_{j} ; M\right)-v_{c}\left(P_{i}, T, w_{j} ;\right.$ Ref $)$.

The pressure $P_{i}$ is in the range $0.1-25.0 \mathrm{MPa}$, with a step of $0.1 \mathrm{MPa}$, and the slit pore width $w_{j}$ is in the range 6-14 $\AA$, with a step of $0.05 \AA$ for benzene slit pores, and in the range $6-12 \AA$, with a step of $0.10 \AA$ for graphene slit pores. The magnitudes $v_{c}\left(P_{i}, T, w_{j} ; M\right)$ and $v_{c}\left(P_{i}, T, w_{j} ;\right.$ Ref $)$ are the volumetric capacities of a benzene or graphene slit pore of width $w_{j}$, obtained with the method $M$ and the reference method, respectively, at pressure $P_{i}$ and temperature T. RMSE (T; M) has the same units as the volumetric capacity $v_{c}, \mathrm{~kg} / \mathrm{L}$, and RMSPE $(\mathrm{T} ; \mathrm{M})$ is a percentage and is in $\%$.

\section{Reference volumetric capacities for benzene and graphene slit pores}

The CCSD(T) method includes accurately the dispersion interactions and therefore, the $\operatorname{CCSD}(\mathrm{T})$ volumetric capacities of benzene slit pores are considered the accurate and reference capacities. To study the effect of the dispersion interactions on benzene slit pores, the capacities obtained with other methods are compared with the $\operatorname{CCSD}(\mathrm{T})$ capacities.

It is not possible nowadays to calculate the $\operatorname{CcSD}(\mathrm{T})$ interaction potential energy curve $\mathrm{V}(\mathrm{z})$ of $\mathrm{H}_{2}$ on graphene, which would allow us to include accurately the dispersion interactions and hence, to calculate accurately the volumetric capacities of graphene slit pores. The alternative is to consider, as the reference capacities for graphene slit pores, the volumetric capacities of the DFT-based method that yield an energy of the lowest physisorbed level of $\mathrm{H}_{2}$ on graphene, $E_{a d s}$, very close to the experimental value.

There are two experimental values of the energy $E_{\text {ads }}$. Mattera et al. [75] measured a value of $-0.04161 \mathrm{eV}$ for the energy of the lowest level of a hydrogen molecule physisorbed on graphite, with an experimental accuracy of $0.00025 \mathrm{eV}$. Costanzo et al. [76] used the Crowell-Brown graphite model [77] to discount the effect of the other graphene layers of graphite, obtaining that the energy should be corrected by $6 \times 10 \mathrm{eV}$ and that the experimental energy of the lowest level of $\mathrm{H}_{2}$ on graphene should be $-0.0476 \mathrm{eV}$.

Matsumoto et al. [78] measured a value of $-0.045 \pm$ $0.005 \mathrm{eV}$ for the adsorption energy of a hydrogen molecule on the basal plane of a nanoneedle, where the crystal structure is very similar to the graphite surface. According to the Crowell-Brown model energy correction [76], the adsorption energy of the lowest level of $\mathrm{H} 2$ on graphene should be $-0.051 \pm 0.005 \mathrm{eV}$. Taking into account all the experimental results, the theoretical adsorption energies, $E_{\text {ads, }}$ that are between -0.056 and $-0.046 \mathrm{eV}$ can be considered correct. Table 1 shows the errors of the adsorption energies on graphene obtained with the DFTbased methods. The errors were calculated respect to the value measured by Matsumoto et al.: $E_{\text {ads,exp }}=-0.051 \pm$ $0.005 \mathrm{eV}$ [78]. The PBE-D2 method yields an adsorption energy of $-0.0507 \mathrm{eV}$, the closest value to the experimental value, $-0.051 \mathrm{eV}$, and hence, the PBE-D2 capacities are selected as the reference capacities of graphene slit pores.

The vdW-DF2 and PBE-XDM methods have adsorption energies of -0.0573 and $-0.0470 \mathrm{eV}$, respectively, close to the respective limits of the experimental error range of the adsorption energy: -0.056 and $-0.046 \mathrm{eV}$. Therefore, it is reasonable to consider that the error of the theoretical capacities of graphene slit pores calculated with any method should be, at least, the difference between the PBE-XDM and vdW-DF2 capacities.

The difference between the PBE-XDM and vdW-DF2 capacities have been calculated using Eqs. (6) and (7). The $M$ and Reference methods were, in this case, the PBE$\mathrm{XDM}$ and vdW-DF2 methods, respectively. The RMSE(T) and RMSPE(T) errors obtained were $0.0038 \mathrm{~kg} / \mathrm{L}$ and $7 \%$ at $80.15 \mathrm{~K}$ and $0.0038 \mathrm{~kg} / \mathrm{L}$ and $27 \%$ at $298.15 \mathrm{~K}$, respectively. These are the smallest errors of the theoretical volumetric capacities of graphene slit pores that should be expected.

Table 1 - Adsorption energies of the lowest level of $\mathrm{H}_{2}$ on graphene, $E_{\text {ads }}$. Error $=E_{\text {ads }}-E_{\text {ads,exp }}$ in eV and Relative error $=100$ Error $/ E_{\text {ads,exp }} \mid$ in \%. $E_{\text {ads }}$ values taken from Ref. [29], except VWN and PW91 values.

\begin{tabular}{llll} 
Method & $E_{a d s}$ & Error & Relative error \\
\hline VWN & -0.0774 & -0.0264 & -51.8 \\
PW91 & -0.0163 & 0.0347 & 68.0 \\
PBE & -0.0062 & 0.0448 & 87.8 \\
PBE + DCACP & -0.0382 & 0.0128 & 25.1 \\
PBE-D2 & -0.0507 & 0.0003 & 0.6 \\
PBE-TS & -0.0610 & -0.0100 & -19.6 \\
PBE-XDM & -0.0470 & 0.0040 & 7.8 \\
vdW-DF & -0.0706 & -0.0196 & -38.4 \\
vdW-DF-C09 & -0.0657 & -0.0147 & -28.8 \\
vdW-DF-cx & -0.0666 & -0.0156 & -30.6 \\
optB86b-vdW & -0.0686 & -0.0176 & -34.5 \\
optB88-vdW & -0.0656 & -0.0146 & -28.6 \\
vdW-DF2 & -0.0573 & -0.0063 & -12.4 \\
rev-vdW-DF2 & -0.0481 & 0.0029 & 5.7 \\
vdW-DF2-C09 & -0.0266 & 0.0244 & 47.8 \\
RVV10 & -0.0520 & -0.0010 & -2.0 \\
\hline
\end{tabular}




\section{Qualitative comparison of the volumetric capacities obtained with the QT model}

The volumetric capacities have been grouped into three groups, taking into account the type of method used to calculate them. The groups are, in increasing order of complexity of the inclusion of the dispersion interactions: a) Capacities obtained with DFT methods that do not include the dispersion interactions, b) capacities obtained with DFT methods that include empirically the dispersion interactions and c) capacities obtained with DFT methods that include the dispersion interactions through non-local functionals. The capacities obtained with the MP2 method are included in the first group. The volumetric capacities of benzene and graphene slit pores of each group are studied and compared in the next subsections as a function of pressure, temperature and pore width.

The reference volumetric capacities are plotted as black solid lines in all the figures. For benzene slit pores the $\operatorname{CCSD}(\mathrm{T})$ capacities are the reference and for graphene slit pores, the PBE-D2 capacities. The volumetric capacities at low temperature, $80.15 \mathrm{~K}$, are larger than at room temperature, $298.15 \mathrm{~K}$, and hence, these capacities are at the top and bottom, respectively, of each figure of the volumetric capacities.

Volumetric capacities obtained with the DFT methods that do not include the dispersion interactions

The volumetric capacities of benzene and graphene slit pores at 80.15 and $298.15 \mathrm{~K}$ obtained with the VWN, PW91 and PBE methods are shown and compared with the reference capacities in Figs. 4 and 5. In the case of benzene slit pores, the MP2 capacities are also studied. These figures show qualitatively the effect of not including the dispersion interactions in the VWN, PW91 and PBE methods and the effect of including them in the MP2 method. At room temperature, $298.15 \mathrm{~K}$, the absolute differences between the reference capacities and the capacities obtained with these methods are smaller than at low temperature, $80.15 \mathrm{~K}$, for both types of pores.

MP2 capacities are relatively close to the reference capacities of benzene slit pores, the $\operatorname{CCSD}(\mathrm{T})$ capacities. The MP2 method includes the dispersion interactions, but not as accurately as the $\operatorname{CCSD}(\mathrm{T})$ method. It does not reproduce well screening effects and sometimes is affected by important errors even in small systems. The VWN, PW91 and PBE methods do not include the dispersion interactions and this can be
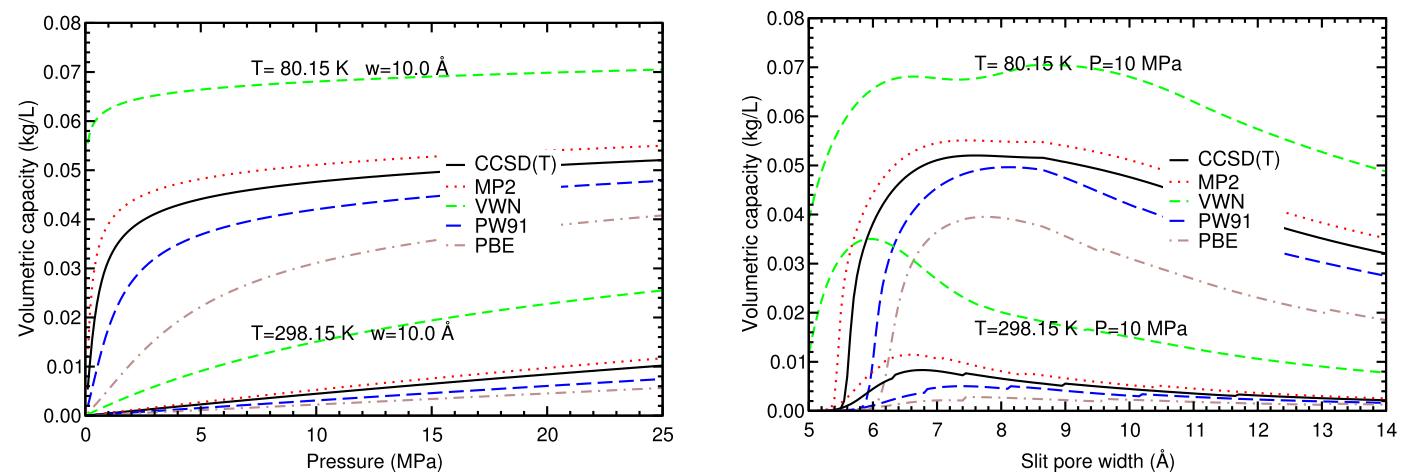

Fig. 4 - Volumetric capacities of benzene slit pores at 80.15 and $298.15 \mathrm{~K}$, as a function of pressure (left panel; width = $10 \AA$ ) and pore width (right panel; $P=10 \mathrm{MPa}$ ), obtained with $\operatorname{CCSD}(\mathrm{T})$, MP2 and DFT methods that do not include dispersion interactions.
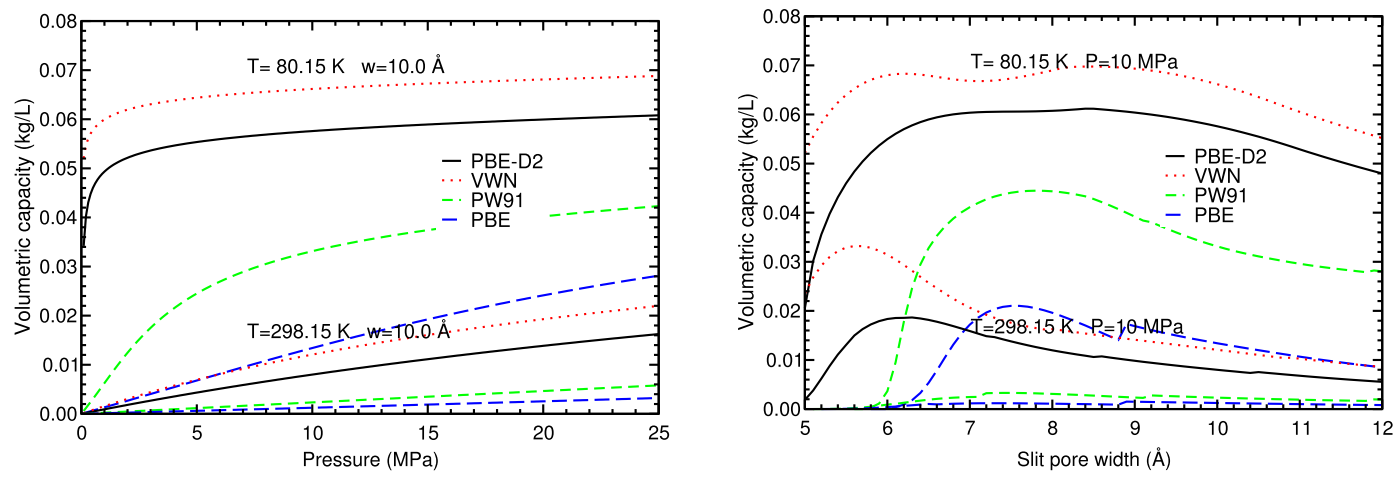

Fig. 5 - Volumetric capacities of graphene slit pores at 80.15 and $298.15 \mathrm{~K}$, as a function of pressure (left panel; width $=10 \AA$ ) and pore width (right panel; $P=10 \mathrm{MPa}$ ), obtained with the reference method and DFT methods that do not include dispersion interactions. 
noticed in their volumetric capacities. The VWN capacities are much larger than the reference capacities of benzene and graphene slit pores, because this method overestimates the $\mathrm{H}_{2}$-surface (benzene or graphene) interaction and therefore, yields higher volumetric capacities. The PBE and PW91 methods underestimate that interaction and yield volumetric capacities lower or much lower than the reference capacities, as can be noticed in Figs. 4 and 5.

The effects of the dispersion interactions have a complex dependence on the pressure (See left panels of Figs. 4 and 5): At low temperatures, $80.15 \mathrm{~K}$, the differences between the reference capacities and the other capacities are larger at low pressures and decrease as the pressure increases. At high temperature, $298.15 \mathrm{~K}$, the dependence on the pressure is the opposite: The differences increases as the pressure increases.

The effects of the dispersion interactions as a function of the pore width are shown in the right panels of Figs. 4 and 5. The MP2 volumetric capacities are similar to the $\operatorname{CCSD}(\mathrm{T})$ capacities as a function of the pore width. However, the VWN, PW91 and $\mathrm{PBE}$ volumetric capacities are very different from the reference capacities as a function of the pore width, for both types of pores, especially the VWN capacities. The differences are much larger at short pore widths than at large pore widths.

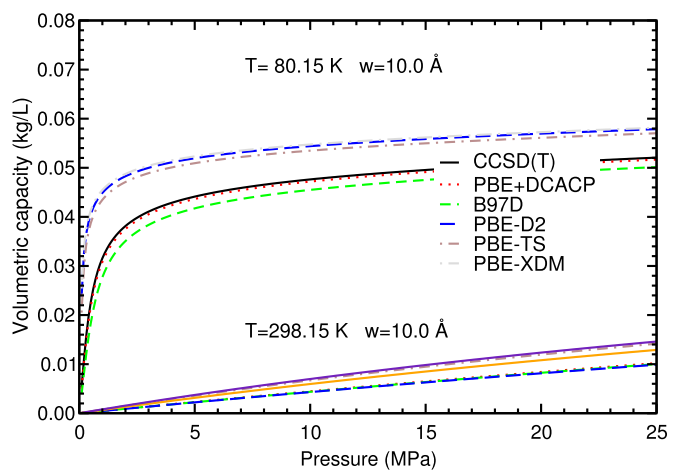

\section{Volumetric capacities obtained with the empirical DFT methods}

The volumetric capacities obtained using DFT methods that include dispersion interactions empirically or semiempirically (PBE + DCACP, B97D, PBE-D2, PBE-TS and PBE-XDM) are plotted and compared in Figs. 6 and 7 with the reference capacities.

The volumetric capacities of the empirical DFT methods are much closer to the reference capacities than the VWN, PW91 and PBE capacities, except the PW91 capacities of benzene slit pores, which are similar or even better than some empirical DFT capacities, as can be noticed by comparing Figs. 4 and 6 . Hence, the introduction of the dispersion interactions in the empirical and semiempirical DFT methods improves, in general, but not in all the comparisons, the volumetric capacities obtained with the VWN, PW91 and PBE methods.

There are some differences between the capacities of benzene and graphene slit pores obtained with each DFT method. For instance, in the case of benzene slit pores, the $\mathrm{PBE}+\mathrm{DCACP}$ and reference capacities are practically identical, while in graphene slit pores are more different. PBE-XDM capacities are somewhat far from the reference capacities in

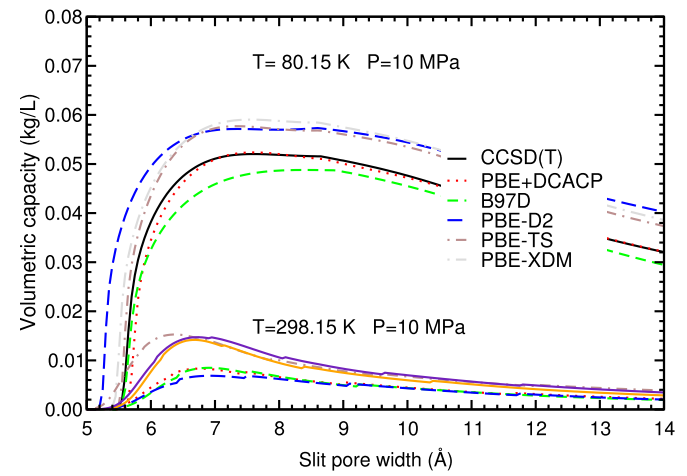

Fig. 6 - Volumetric capacities of benzene slit pores at 80.15 and $298.15 \mathrm{~K}$, as a function of pressure (left panel; width = $10 \AA$ ) and pore width (right panel; $P=10 \mathrm{MPa}$ ), obtained with $\mathrm{CCSD}(\mathrm{T})$ and $\mathrm{DFT}$ methods that include dispersion interactions empirically or semiempirically.
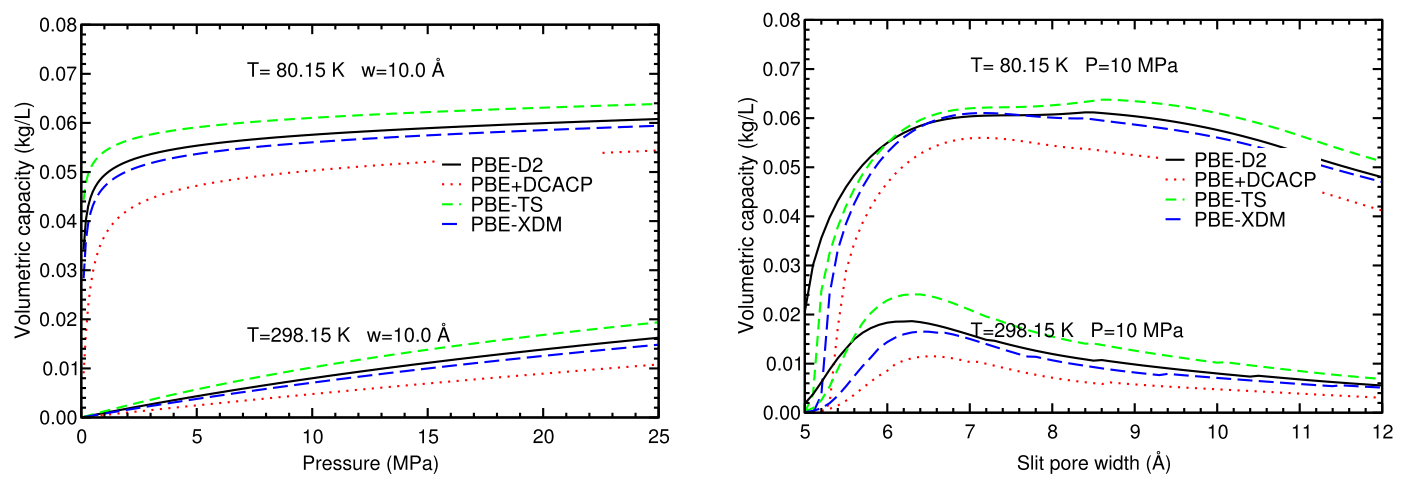

Fig. 7 - Volumetric capacities of graphene slit pores at 80.15 and $298.15 \mathrm{~K}$, as a function of pressure (left panel; width = $10 \AA$ ) and pore width (right panel; $P=10 \mathrm{MPa}$ ), obtained with the reference method and DFT methods that include dispersion interactions empirically or semiempirically. 
benzene slit pores, while they are very similar to the graphene slit pore reference capacities. The B97D capacities are close to the $\operatorname{CCSD}(\mathrm{T})$ reference capacities.

The differences between the reference and the empirical DFT capacities are, in general, larger at narrow pore widths than at wide pore widths (See right panels of Figs. 6 and 7).
This means that the inclusion of the dispersion interactions is more important for narrow pores than for wide pores. The $\operatorname{CCSD}(\mathrm{T})$ and PBE + DCACP capacities agree very well at any pore width of benzene slit pores. The agreement between the $\operatorname{CCSD}(\mathrm{T})$ and B97D capacities is also important, but less perfect.
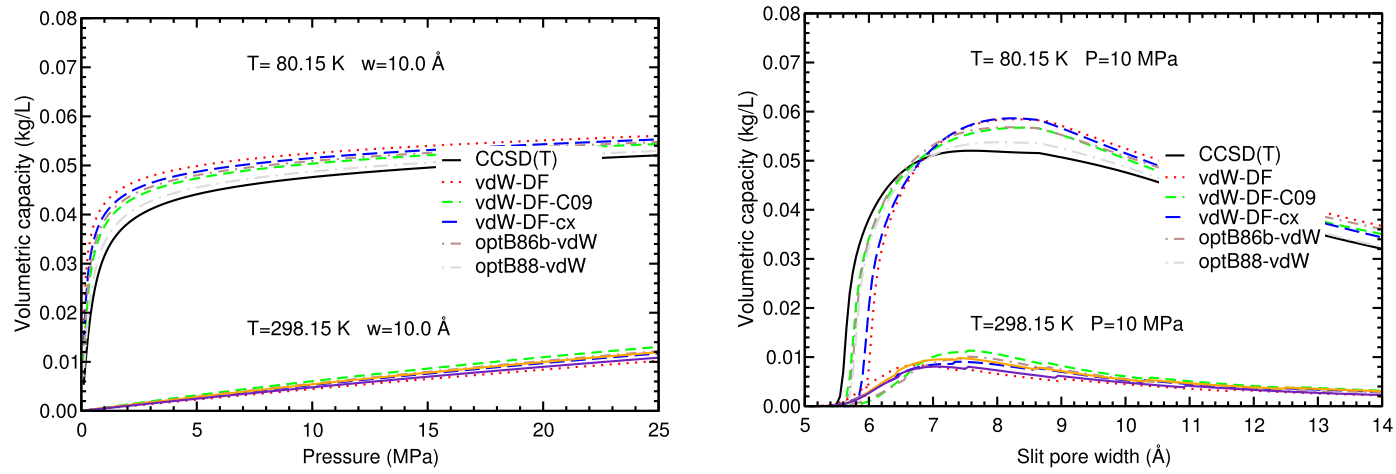

Fig. 8 - Volumetric capacities of benzene slit pores at 80.15 and $298.15 \mathrm{~K}$, as a function of pressure (left panel; width = $10 \AA)$ and pore width (right panel; $P=10 \mathrm{MPa}$ ), obtained with $\operatorname{CCSD}(\mathrm{T})$ and vdW-DF methods.
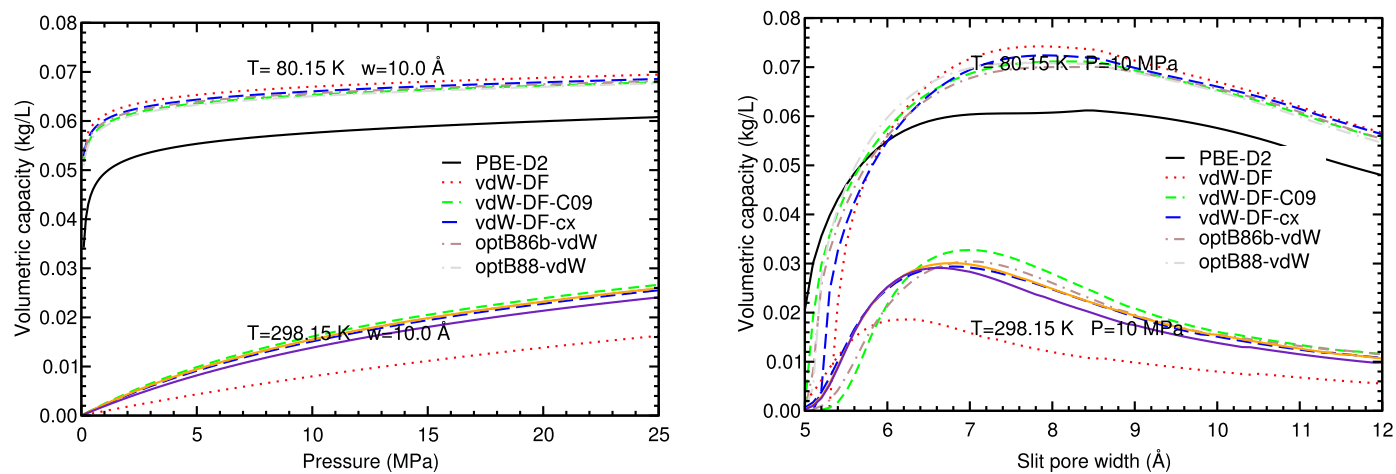

Fig. 9 - Volumetric capacities of graphene slit pores at 80.15 and $298.15 \mathrm{~K}$, as a function of pressure (left panel; width = $10 \AA)$ and pore width (right panel; $P=10 \mathrm{MPa}$ ), obtained with the reference method and vdW-DF methods.
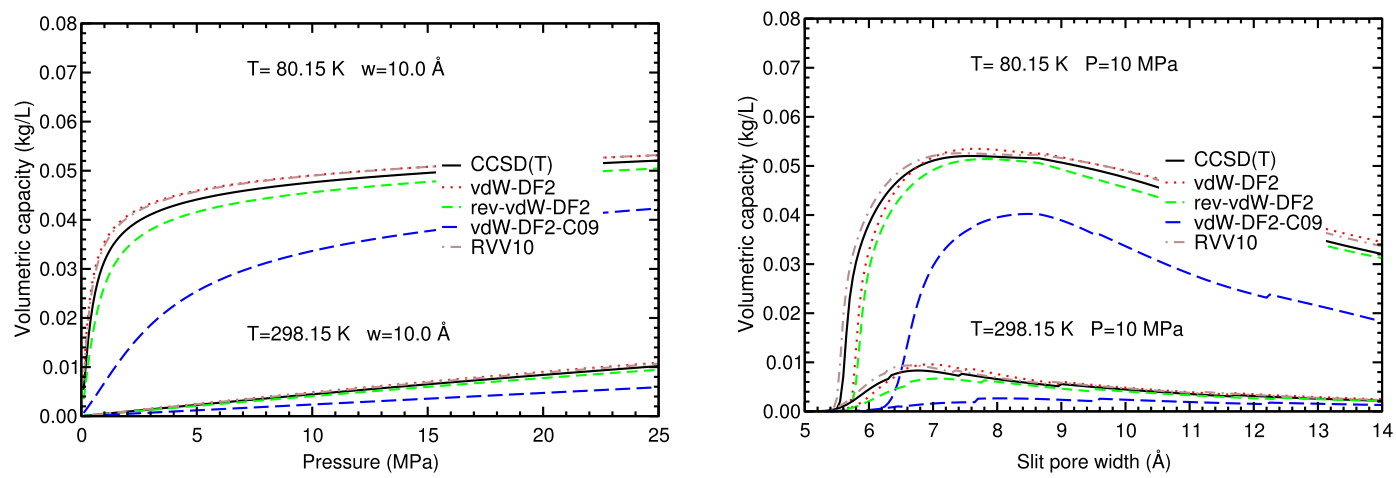

Fig. 10 - Volumetric capacities of benzene slit pores at 80.15 and $298.15 \mathrm{~K}$, as a function of pressure (left panel; width = $10 \AA)$ and pore width (right panel; $P=10 \mathrm{MPa}$ ), obtained with $\operatorname{CCSD}(\mathrm{T})$ and vdW-DF2 methods. 
At $80.15 \mathrm{~K}$ the differences between the reference capacities and the capacities obtained with other methods are larger at low pressures than at high pressures and decrease as the pressure increases. At $298.15 \mathrm{~K}$ the trend of the differences is different: The differences increase as the pressure increases.

\section{Volumetric capacities obtained with the non-local DFT methods}

The volumetric capacities obtained with the non-local DFT functionals have been divided into two subgroups: vdW-DF capacities in Figs. 8 and 9 and vdW-DF2 and RVV10 capacities in Figs. 10 and 11. These methods include the dispersion interactions at the most complex level analyzed in this paper.

The results of the vdW-DF capacities depend on the type of pore. In the case of benzene slit pores, the vdW-DF capacities are closer to the reference capacities than the VWN, PW91, PBE and empirical DFT capacities (See Figs. 4, 6 and 8). The optB88-vdW capacities are very close. However, the vdW-DF capacities of graphene slit pores are very different from the reference capacities (See Fig. 9) and the empirical DFT capacities are as close to the reference capacities as the vdW-DF capacities or even closer, as can be observed by comparing Figs. 7 and 9.

The vdW-DF functionals include the dispersion interactions, but their adsorption energies of the first level of $\mathrm{H}_{2}$ on graphene, $E_{a d s}$, have an important relative error, between -39 and $-28 \%$ (See Table 1 ). This means that these methods do not reproduce well the binding energy region of $\mathrm{V}(\mathrm{z})$ on graphene and the result is that their capacities are very different from the reference capacities of graphene slit pores.

The vdW-DF2, rev-vdW-DF2 and RVV10 capacities are similar to the reference capacities of benzene and graphene slit pores, as can be seen in Figs. 10 and 11. The vdW-DF2-C09 capacities, however, are very different for both types of pores. This means that the dispersion interactions in graphitic-like surfaces (benzene and graphene) are not included correctly in vdW-DF2-C09 and are included accurately in the functionals vdW-DF2, rev-vdW-DF2 and RVV10.

The volumetric capacities as a function of the pressure obtained with non-local functionals are close or very close to the reference capacities. However, the capacities as a function of the pore width are less closer to the reference capacities, especially at narrow pores: 5-6 ^ (See right panels of Figs. 8-11)). This result was also observed in the VWN, PW91, PBE and empirical DFT capacities. Hence, also for the non-local functionals the effects of the dispersion interactions are more important at narrow pores than at wide pores.

\section{Quantitative comparison: errors of the volumetric capacities obtained with the QT model}

A qualitative comparison of the effects of including the dispersion interactions in the DFT methods has been discussed in the above section. In this section the average errors of the capacities obtained with the methods are presented and discussed. The purpose of these calculations is a) to provide not only a value of the theoretical volumetric capacity obtained with a method and the QT model, but also the error of the theoretical capacity and b) to find out which are the most accurate DFT methods to simulate the volumetric capacities of nanoporous carbons, simulated as benzene and graphene slit pores. The values of the absolute and relative errors depend on the type of pore.

\section{Errors of the volumetric capacities of benzene slit pores}

The average errors of the volumetric capacities of benzene slit pores obtained with each method are shown in Table 2. A general trend is that the absolute error at $\mathrm{T}=298.15 \mathrm{~K}$ is smaller than at $\mathrm{T}=80.15 \mathrm{~K}$ (See Table 2). However, this reduction of the absolute error from 80.15 to $298.15 \mathrm{~K}$ is a mathematical artifact that has to do with the fact that the numerical values of the volumetric capacities decrease as the temperature increases, as can be seen in Figs. 4, 6, 8 and 10. This was the reason to calculate and to study also the relative error. The relative error increases from 80.15 to $298.15 \mathrm{~K}$, except for B97D, where it remains constant.

The smallest errors correspond to six DFT-based methods: PBE + DCACP, B97D, optB88-vdW, vdW-DF2, rev-vdW-DF2 and RVV10. The errors of the volumetric capacities obtained with the other methods are high or very high. The average relative errors of the volumetric capacities at $80.15 \mathrm{~K}$ are in the range
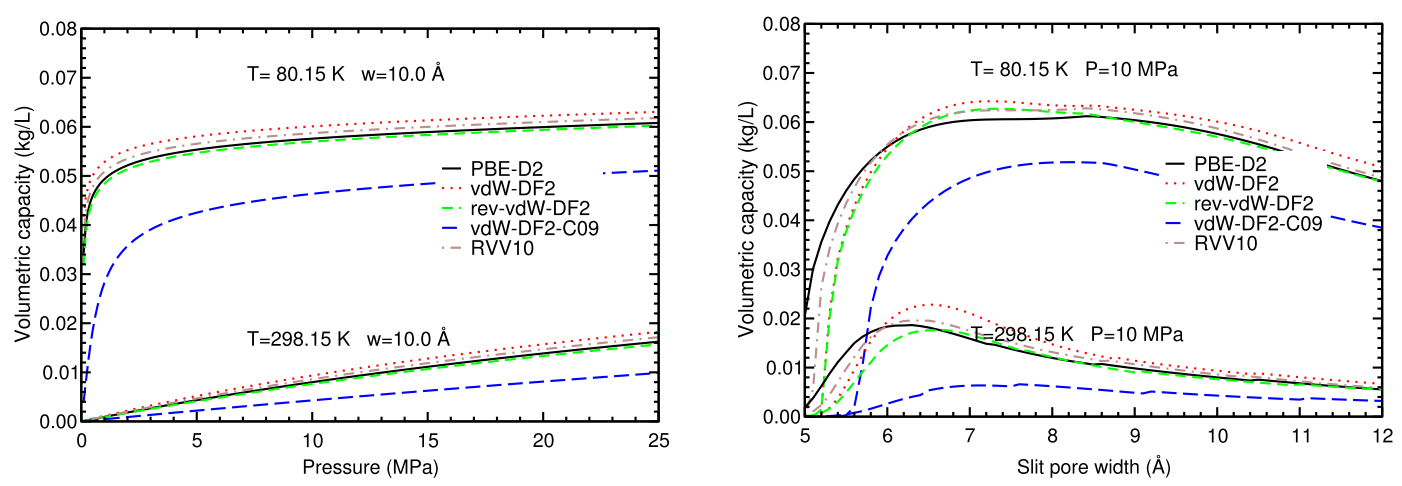

Fig. 11 - Volumetric capacities of graphene slit pores at 80.15 and $298.15 \mathrm{~K}$, as a function of pressure (left panel; width $=10 \AA$ ) and pore width (right panel; $\mathrm{P}=10 \mathrm{MPa}$ ), obtained with the reference method and vdW-DF2 methods. 
Table 2 - Average errors of volumetric capacities of benzene slit pores. RMSE in kg/L and RMSPE in \%.

\begin{tabular}{|c|c|c|c|c|}
\hline \multirow[b]{2}{*}{ Method M } & \multicolumn{2}{|c|}{$\mathrm{T}=80.15 \mathrm{~K}$} & \multicolumn{2}{|c|}{$\mathrm{T}=298.15 \mathrm{~K}$} \\
\hline & RMSE (T; M) & RMSPE (T; M) & RMSE (T; M) & RMSPE (T; M) \\
\hline MP2 & 0.0038 & 15 & 0.0018 & 28 \\
\hline VWN & 0.0199 & 105 & 0.0133 & 335 \\
\hline PW91 & 0.0069 & 20 & 0.0023 & 32 \\
\hline PBE & 0.0173 & 44 & 0.0040 & 57 \\
\hline $\mathrm{PBE}+\mathrm{DCACP}$ & 0.0008 & 2 & 0.0004 & 7 \\
\hline B97D & 0.0033 & 9 & 0.0007 & 9 \\
\hline PBE-D2 & 0.0079 & 47 & 0.0038 & 70 \\
\hline PBE-TS & 0.0063 & 30 & 0.0029 & 46 \\
\hline PBE-XDM & 0.0077 & 40 & 0.0038 & 64 \\
\hline vdW-DF & 0.0066 & 26 & 0.0026 & 44 \\
\hline vdW-DF-C09 & 0.0034 & 12 & 0.0014 & 25 \\
\hline vdW-DF-cx & 0.0051 & 16 & 0.0020 & 31 \\
\hline optB86b-vdW & 0.0041 & 17 & 0.0016 & 29 \\
\hline optB88-vdW & 0.0016 & 5 & 0.0007 & 12 \\
\hline vdW-DF2 & 0.0023 & 10 & 0.0008 & 13 \\
\hline rev-vdW-DF2 & 0.0024 & 7 & 0.0012 & 16 \\
\hline vdW-DF2-C09 & 0.0188 & 47 & 0.0043 & 57 \\
\hline RVV10 & 0.0016 & 6 & 0.0006 & 10 \\
\hline
\end{tabular}

$2-10 \%$ and at $298.15 \mathrm{~K}$ are in the range $7-16 \%$ obtained with these six methods. The smallest errors correspond to the $\mathrm{PBE}+$ DCACP volumetric capacities.

According to the errors of the volumetric capacities showed in Table 2, several DFT-based methods outperform, on average, to the MP2 method: PBE + DCACP, B97D, vdW-DFC09, optB88-vdW, RVV10, vdW-DF2 and rev-vdW-DF2. Some methods that do not include the dispersion interactions have smaller average errors than other methods that include the dispersion interactions empirically or through non-local functionals: PW91 performs, on average, better than PBE-D2, PBE-TS and PBE-XDM, vdW-DF and vdW-DF2-C09. Some methods that include the dispersion interactions empirically have smaller average errors than other methods that include them through non-local functionals: PBE + DCACP and B97D perform better or much better than vdW-DF, vdW-DF-C09, vdW-DF-cx, optB86b-vdW and vdW-DF2-C09. Finally, the optB88-vdW method of the vdW type has much smaller average errors than the vdW-DF2-C09 method, and slightly smaller average errors than the vdW-DF2 and rev-vdW-DF2 methods. Hence, a higher complexity of the inclusion of the dispersion interactions in a DFT-based method does not guarantee necessarily more accurate volumetric capacities. Each DFT-based method must be tested.

\section{Errors of the volumetric capacities of graphene slit pores}

The average errors of the volumetric capacities of graphene slit pores obtained with each method are shown in Table 3. The errors were calculated respect to the reference capacities, the PBE-D2 ones, and the minimum errors calculated in section 4 were applied to the capacities obtained with any method. The smallest errors of the capacities in Table 3 correspond to the methods PBE-D2, PBE-TS, PBE-XDM, vdWDF2, rev-vdW-DF2 and RVV10 and are equal to the minimum errors, except the relative error of PBE-TS at 298.15 K. As in the

Table 3 - Average errors of volumetric capacities of graphene slit pores. RMSE in kg/L and RMSPE in \%.

\begin{tabular}{lllll} 
& \multicolumn{2}{c}{$\mathrm{T}=80.15 \mathrm{~K}$} & & $\mathrm{~T}=298.15 \mathrm{~K}$ \\
\hline Method M & RMSE (T; M) & RMSPE (T; M) & RMSE (T; M) & RMSPE (T; M) \\
VWN & 0.0084 & 15 & 0.0051 & 47 \\
PW91 & 0.0249 & 46 & 0.0104 & 75 \\
PBE & 0.0428 & 76 & 0.0120 & 88 \\
PBE + DCACP & 0.0072 & 14 & 0.0048 & 40 \\
PBE-D2 & 0.0038 & 7 & 0.0038 & 27 \\
PBE-TS & 0.0038 & 7 & 0.0038 & 31 \\
PBE-XDM & 0.0038 & 7 & 0.0038 & 27 \\
vdW-DF & 0.0105 & 19 & 0.0111 & 124 \\
vdW-DF-C09 & 0.0084 & 15 & 0.0092 & 99 \\
vdW-DF-cx & 0.0092 & 16 & 0.0098 & 109 \\
optB86b-vdW & 0.0080 & 15 & 0.0094 & 104 \\
optB88-vdW & 0.0083 & 15 & 0.0081 & 82 \\
vdW-DF2 & 0.0038 & 7 & 0.0038 & 27 \\
rev-vdW-DF2 & 0.0038 & 7 & 0.0038 & 27 \\
vdW-DF2-C09 & 0.0124 & 23 & 0.0074 & 52 \\
RVV10 & 0.0038 & 7 & 0.0038 & 27 \\
\hline
\end{tabular}


case of benzene slit pores, the capacities at room temperature have larger relative errors than the capacities at low temperature, $80.15 \mathrm{~K}$.

The results in Table 3 show that the average errors of the capacities of graphene slit pores obtained with empirical DFT methods are smaller than the average errors obtained with some complex methods that include the dispersion interactions, such as the vdW-DF and vdW-DF2-C09 methods. The empirical DFT and vdW-DF2 methods, except the vdWDF2-C09 method, have similar average errors. This, again, indicates that the complexity of the inclusion of the dispersion interactions in a DFT-based method is not proportional to the accuracy of the volumetric capacities calculated with that method. As in the case of benzene slit pores, each method must be tested.

\section{Conclusions}

Calculations of the volumetric capacities of nanoporous carbons simulated as benzene and graphene slit pores and using $\operatorname{CCSD}(\mathrm{T}), \mathrm{MP} 2$ and 17 DFT-based methods have been carried out. Three DFT-based methods do not include the dispersion interactions and 14 methods include them at different levels of complexity.

The volumetric capacities of benzene slit pores have been compared with the capacities obtained with the $\operatorname{CCSD}(\mathrm{T})$ method, because this method describes accurately the dispersion interactions, and the volumetric capacities of graphene slit pores have been compared with experimental results of the adsorption of $\mathrm{H}_{2}$ on graphene. It is found from these comparisons that a higher complexity of the inclusion of the dispersion interactions in a DFT-based method does not imply necessarily more accurate volumetric capacities. Each DFT-based method must be tested, regardless of its complexity. The comparisons show that the most accurate volumetric capacities of benzene and graphene slit pores are obtained with the DFT methods vdW-DF2, rev-vdW-DF2 and RVV10. The estimated relative errors of the capacities obtained with these methods are in the range $7-10 \%$ at $80.15 \mathrm{~K}$ and $27 \%$ at $298.15 \mathrm{~K}$. These DFT-based methods should be used to simulate accurately the volumetric storage capacities of nanoporous carbons.

\section{Acknowledgment}

This work was supported by MINECO of Spain (Grant MAT2014-54378-R), Junta de Castilla y León (Grants VA050U14 and VA124G18) and the University of Valladolid. The facilities provided by Centro de Proceso de Datos - Parque Científico of the University of Valladolid are also acknowledged.

\section{R E F E R E N C E S}

[1] Ismail M, Mustafa NS, Ali NA, Sazelee NA, Yahya MS. The hydrogen storage properties and catalytic mechanism of the
$\mathrm{CuFe}_{2} \mathrm{O}_{4}$-doped $\mathrm{MgH}_{2}$ composite system. Int J Hydrogen Energy 2019;44:318-24.

[2] Wang $\mathrm{K}, \mathrm{Wu}$ G, Cao H, Li H, Zhao X. Improved reversible dehydrogenation properties of $\mathrm{MgH}_{2}$ by the synergetic effects of graphene oxide-based porous carbon and $\mathrm{TiCl}_{3}$. Int $\mathrm{J}$ Hydrogen Energy 2018;43:7440-6.

[3] Yahya MS, I M. Synergistic catalytic effect of $\mathrm{SrTiO}_{3}$ and Ni on the hydrogen storage properties of $\mathrm{MgH}_{2}$. Int J Hydrogen Energy 2018;43:6244-55.

[4] Pighin SA, Coco B, Troiani H, Castro FJ, Urretavizcaya G. Effect of additive distribution in $\mathrm{H}_{2}$ absorption and desorption kinetics in $\mathrm{MgH}_{2}$ milled with $\mathrm{NbH}_{0.9}$ or $\mathrm{NbF}_{5}$. Int $J$ Hydrogen Energy 2018;43:7430-9.

[5] Yahya MS, Sulaiman NN, Mustafa NS, Yap FAH, Ismail M. Improvement of hydrogen storage properties in $\mathrm{MgH}_{2}$ catalysed by $\mathrm{K}_{2} \mathrm{NbF}_{7}$. Int J Hydrogen Energy 2018;43:14532-40.

[6] Korablov D, Besenbacher F, Jensen TR. Kinetics and thermodynamics of hydrogenation-dehydrogenation for $\mathrm{Mg}$ $25 \% \mathrm{TM}$ ( $\mathrm{TM}=\mathrm{Ti}, \mathrm{Nb}$ or $\mathrm{V}$ ) composites synthesized by reactive ball milling in hydrogen. Int J Hydrogen Energy 2018;43:16804-14.

[7] Sazelee NA, Idris NH, Din MFM, Mustafa NS, Ali NA, Yahya MS, et al. Synthesis of $\mathrm{BaFe}_{12} \mathrm{O}_{19}$ by solid state method and its effect on hydrogen storage properties of $\mathrm{MgH}_{2}$. Int J Hydrogen Energy 2018;43:20853-60.

[8] Yap FAH, Sulaiman NN, Ismail M. Understanding the dehydrogenation properties of $\mathrm{MgH}_{2}$ catalysed by $\mathrm{Na}_{3} \mathrm{AlF}_{6}$. Int J Hydrogen Energy 2018. https://doi.org/10.1016/ j.ijhydene.2018.02.073.

[9] Sulaiman NN, Ismail M. Effects of $\mathrm{TiF}_{3}$ addition on the hydrogen storage properties of $4 \mathrm{MgH}_{2}+\mathrm{Cd}$ composite. Int J Hydrogen Energy 2018. https://doi.org/10.1016/ j.ijhydene.2018.03.095.

[10] Zou L, Zhou HC. Hydrogen storage in metal-organic frameworks. Nanostructured materials for next-generation energy storage and conversion - hydrogen production, storage, and utilization. Berlin, Heidelberg: Springer-Verlag; 2017. p. 143-70. chap. 5.

[11] Xia L, Liu Q. Adsorption of $\mathrm{H}_{2}$ on aluminum-based metalorganic frameworks: a computational study. Comput Mater Sci 2017;126:176-81.

[12] Gygi D, Bloch ED, Mason JA, Hudson MR, González MI, Siegelman RL, et al. Hydrogen storage in the expanded pore metal-organic frameworks $\mathrm{M}_{2}$ (dobpdc) ( $\mathrm{M}=\mathrm{Mg}, \mathrm{Mn}$, Fe, Co, Ni, Zn). Chem Mater 2016;28:1128-38.

[13] Zhu G, Sun Q. Recent advances in computational studies of organometallic sheets: magnetism, adsorption and catalysis. Comput Mater Sci 2016;112:492-502.

[14] Diercks CS, Yaghi OM. The atom, the molecule, and the covalent organic framework. Science 2017;355. eaal1585.

[15] He T, Pachfule P, Wu H, Xu Q Chen P. Hydrogen carriers. Nature Reviews Materials 2016;1:16059.

[16] Li ZP, Zhi YF, Feng X, Ding XS, Zou YC, Liu XM, et al. An azine-linked covalent organic framework: synthesis, characterization and efficient gas storage. Chem Eur J 2015;21:12079-84.

[17] Li XD, Zang HP, Wang JT, Wang JF, Zhang H. Design of tetraphenyl silsesquioxane based covalent-organic frameworks as hydrogen storage materials. J Mater Chem A 2014;2:18554-61.

[18] Lu W. Strategies for hydrogen storage in porous organic polymers. Nanostructured materials for next-generation energy storage and conversion hydrogen production, storage, and utilization. Springer; 2017. p. 203-23. chap. 7.

[19] Lu XL, Zhou TY, Wu D, Wen Q, Zhao X, Li Q et al. A triptycene-based porous organic polymer that exhibited high 
hydrogen and carbon dioxide storage capacities and excellent $\mathrm{CO}_{2} / \mathrm{N}_{2}$ selectivity. Chin J Chem 2015;33:539-44.

[20] Liu GL, Wang YX, Shen CJ, Ju ZF, Yuan DQ. A facile synthesis of microporous organic polymers for efficient gas storage and separation. J Mater Chem A 2015;3:3015-58.

[21] Xu Y, Jin S, Xu H, Nagai A, Jiang D. Conjugated microporous polymers: design, synthesis and application. Chem Soc Rev 2013;42:8012-31.

[22] Blankenship TS, Balahmar N, Mokaya R. Oxygen-rich microporous carbons with exceptional hydrogen storage capacity. Nat Commun 2017;8:1545.

[23] Blankenship TS, Mokaya R. Cigarette butt-derived carbons have ultra-high surface area and unprecedented hydrogen storage capacity. Energy Environ Sci 2017;10:2552-62.

[24] Mortazavi SZ, Reyhani A, Mirershadi S. Hydrogen storage properties of multi-walled carbon nanotubes and carbon nano-onions grown on single and bi-catalysts including Fe, $\mathrm{Mo}, \mathrm{Co}$ and Ni supported by $\mathrm{MgO}$. Int J Hydrogen Energy 2017;42:24885-96.

[25] Sethia G, Sayari A. Activated carbon with optimum pore size distribution for hydrogen storage. Carbon 2016;99:289-94.

[26] Wróbel-Iwaniec I, Díez N, Gryglewicz G. Chitosan-based highly activated carbons for hydrogen storage. Int J Hydrogen Energy 2015;40:5788-96.

[27] Krasnov PO, Shkaberina GS, Kuzubov AA, Kovaleva EA. Molecular hydrogen sorption capacity of D-schwarzites. Appl Surf Sci 2017;416:766-71.

[28] Wang FD, Zhang T, Hou XY, Zhang WQ Tang SW, Sun H, et al. Li-decorated porous graphene as a high-performance hydrogen storage material: a first-principles study. Int J Hydrogen Energy 2017;42:10099-108.

[29] Cabria I, López MJ, Alonso JA. Searching for DFT-based methods that include dispersion interactions to calculate the physisorption of $\mathrm{H}_{2}$ on benzene and graphene. J Chem Phys 2017;146:214104.

[30] Ozturk Z, Baykasoglu C, Kirca M. Sandwiched graphenefullerene composite: a novel 3-D nanostructured material for hydrogen storage. Int J Hydrogen Energy 2016;41:6403-11.

[31] Avdeenkov A, Bodrenko I, Bessarabov D, Bibikov A, Nikolaev A, Taran M, et al. Thermodynamical model for hydrogen storage capacity in carbon nanostructures. Int J Hydrogen Energy 2015;40:4184-93.

[32] Bartolomei M, Carmona-Novillo E, Giorgi G. First principles investigation of hydrogen physical adsorption on graphynes layers. Carbon 2015;95:1076-81.

[33] Granja A, Alonso JA, Cabria I, López MJ. Competition between molecular and dissociative adsorption of hydrogen on palladium clusters deposited on defective graphene. RSC Adv 2015;5:47945-53.

[34] Lebon A, Carrete J, Gallego LJ, Vega A. Ti-decorated zigzag graphene nanoribbons for hydrogen storage. A van der Waals-corrected density-functional study. Int J Hydrogen Energy 2015;40:4960-8.

[35] Robledo CB, Rojas MI, Cámara O, Leiva EPM. First-principles studies concerning optimization of hydrogen storage in nanoporous reduced graphite oxide. Int J Hydrogen Energy 2014;39:4396-403.

[36] Alonso JA, Cabria I, López MJ. Simulation of hydrogen storage in porous carbons. J Mater Res 2013;28:589-604.

[37] Gracia-Espino E, López-Urías F, Terrones H, Terrones M. Novel nanocarbons for adsorption. Novel carbons adsorbents. Oxford: Elsevier; 2012. p. 3-34. chap. 1.

[38] López MJ, Cabria I, Alonso JA. Simulated porosity and electronic structure of nanoporous carbons. J Chem Phys 2011;135:104706.

[39] Ihm Y, Cooper VR, Peng L, Morris JR. The influence of dispersion interactions on the hydrogen adsorption properties of expanded graphite. J Phys Condens Matter 2012;24:424205.

[40] Mpourmpakis G, Froudakis GE. Assessing the density functional theory in the hydrogen storage problem. J Nanosci Nanotechnol 2008;8:3091-6.

[41] Cabria I, López MJ, Alonso JA. Simulation of the hydrogen storage in nanoporous carbons with different pore shapes. Int J Hydrogen Energy 2011;36:10748-59.

[42] Cabria I, López MJ, Alonso JA. Hydrogen storage capacities of nanoporous carbon calculated by density functional and Møller-Plesset methods. Phys Rev B 2008;78:075415.

[43] Cabria I, López MJ, Alonso JA. Hydrogen storage in pure and Li-doped carbon nanopores: combined effects of concavity and doping. J Chem Phys 2008;128:144704.

[44] Cabria I, López MJ, Alonso JA. The optimum average nanopore size for hydrogen storage in carbon nanoporous materials. Carbon 2007;45:2649-58.

[45] Patchkovskii S, Tse JS, Yurchenko SN, Zhechkov L, Heine T, Seifert G. Graphene nanostructures as tunable storage media for molecular hydrogen. Proc Natl Acad Sci USA 2005;102:10439-44.

[46] Heine T, Zhechkov L, Seifert G. Hydrogen storage by physisorption on nanostructured graphite platelets. Phys Chem Chem Phys 2004;6:980-4.

[47] Čížek J. On the correlation problem in atomic and molecular systems. Calculation of wavefunction components in Urselltype expansion using quantum-field theoretical methods. J Chem Phys 1966;45:4256-66.

[48] Ahn C, Purewal J. Storage materials based on hydrogen physisorption. Hydrogen storage technology. Materials and applications. CRC Press; 2016. p. 213-38. chap. 7.

[49] Gross KJ, Carrington KR, Barcelo S, Karkamkar A, Purewal J, Parrilla P. Recommended best practices for the characterization of storage properties of hydrogen storage materials. Report to the Department of Energy Office of Energy Efficiency and Renewable Energy Hydrogen Storage Program under National Renewable Energy Laboratory Contract No. 147388. H2 Technology Consulting LLC; 2012. http://energy.gov/eere/fuelcells/downloads/recommendedbest-practices-characterization-storage-propertieshydrogen-0. [Accessed 6 March 2019]. http://energy.gov/ sites/prod/files/2014/03/f12/best_practices_hydrogen _storage.pdf.

[50] Broom DP. Hydrogen sorption properties of materials. In: Hydrogen storage materials. The characterisation of their storage properties. London: Springer-Verlag; 2011. p. 61-116. chap. 3.

[51] Park MS, Lee SE, Kim MI, Lee YS. $\mathrm{CO}_{2}$ adsorption characteristics of slit-pore shaped activated carbon prepared from cokes with high crystallinity. Carbon Lett 2015;16:45-50.

[52] Møller C, Plesset MS. Note on an approximation treatment for many-electron systems. Phys Rev 1934;46:618-22.

[53] Perdew JP, Burke K, Ernzerh of M. Generalized gradient approximation made simple. Phys Rev Lett 1996;77:3865-8.

[54] Perdew JP, Burke K, Ernzerhof M. Errata: generalized gradient approximation made simple. Phys Rev Lett 1997;78:1396.

[55] Vosko SH, Wilk L, Nusair M. Accurate spin-dependent electron liquid correlation energies for local spin density calculations: a critical analysis. Can J Phys 1980;58:1200-11.

[56] Perdew JP, Wang Y. Accurate and simple analytic representation of the electron-gas correlation energy. Phys Rev B 1992;45:13244.

[57] von Lilienfeld OA, Tavernelli I, Rothlisberger U, Sebastiani D. Optimization of effective atom centered potentials for London dispersion forces in density functional theory. Phys Rev Lett 2004;93:153004. 
[58] von Lilienfeld OA, Tavernelli I, Rothlisberger U, Sebastiani D. Performance of optimized atom-centered potentials for weakly bonded systems using density functional theory. Phys Rev B 2005;71:195119.

[59] Tapavicza E, Lin IC, von Lilienfeld OA, Tavernelli I, CoutinhoNeto MD, Rothlisberger U. Weakly bonded complexes of aliphatic and aromatic carbon compounds described with dispersion corrected density functional theory. J Chem Theory Comput 2007;3:1673-9.

[60] Lin I, Coutinho-Neto MD, Felsenheimer C, von Lilienfeld OA, Tavernelli I, Rothlisberger U. Library of dispersion-corrected atom-centered potentials for generalized gradient approximation functionals: elements $\mathrm{H}, \mathrm{C}, \mathrm{N}, \mathrm{O}, \mathrm{He}, \mathrm{Ne}, \mathrm{Ar}$, and Kr. Phys Rev B 2007;75:205131.

[61] Grimme S. Semiempirical GGA-type density functional constructed with a long-range dispersion correction. J Comput Chem 2006;27:1787-99.

[62] Becke AD. Density-functional thermochemistry. V. Systematic optimization of exchange-correlation functionals. J Chem Phys 1997;107:8554-60.

[63] Tkatchenko A, Scheffler M. Accurate molecular van der Waals interactions from ground-state electron density and free-atom reference data. Phys Rev Lett 2009;102:073005.

[64] Becke AD, Johnson ER. Exchange-hole dipole moment and the dispersion interaction revisited. J Chem Phys 2007;127:154108.

[65] de la Roza AO, Johnson ER. Van der Waals interactions in solids using the exchange-hole dipole moment model. J Chem Phys 2012;136:174109.

[66] Dion M, Rydberg H, Schröder E, Langreth DC, Lundqvist BI. Van der Waals density functional for general geometries. Phys Rev Lett 2004;92:246401.

[67] Klimeš J, Bowler DR, Michaelides A. Chemical accuracy for the van der Waals density functional. J Phys Condens Matter 2010;22:022201.
[68] Klimeš J, Bowler DR, Michaelides A. Van der Waals density functionals applied to solids. Phys Rev B 2011;83:195131.

[69] Berland K, Hyldgaard P. Exchange functional that tests the robustness of the plasmon description of the van der Waals density functional. Phys Rev B 2014;89:035412.

[70] Cooper VR. Van der Waals density functional: an appropriate exchange functional. Phys Rev B 2010;81:161104(R).

[71] Lee K, Murray ED, Kong L, Lundqvist BI, Langreth DC. Higheraccuracy van der Waals density functional. Phys Rev B 2010;82:081101(R).

[72] Hamada I, Otani M. Comparative van der Waals densityfunctional study of graphene on metal surfaces. Phys Rev B 2010;82:153412.

[73] Hamada I. van der Waals density functional made accurate. Phys Rev B 2014;89:121103(R).

[74] Sabatini R, Gorni T, de Gironcoli S. Nonlocal van der Waals density functional made simple and efficient. Phys Rev B 2013;87:041108(R).

[75] Mattera L, Rosatelli R, Salvo C, Tommasini F, Valbusa U, Vidali G. Selective adsorption of ${ }^{1} \mathrm{H}_{2}$ and ${ }^{2} \mathrm{H}_{2}$ on the (0001) graphite surface. Surf Sci 1980;93:515-25.

[76] Costanzo F, Silvestrelli PL, Ancilotto F. Physisorption, diffusion, and chemisorption pathways of $\mathrm{H}_{2}$ molecule on graphene and on $(2,2)$ carbon nanotube by first principles calculations. J Chem Theory Comput 2012;8:1288-94.

[77] Crowell AD, Brown JS. Laterally averaged interaction potentials for ${ }^{1} \mathrm{H}_{2}$ and ${ }^{2} \mathrm{H}_{2}$ on the (0001) graphite surface. Surf Sci 1982;123:296-304.

[78] Matsumoto T, Neo Y, Mimura H, Tomita M. Determining the physisorption energies of molecules on graphene nanostructures by measuring the stochastic emissioncurrent fluctuation. Phys Rev E 2008;77:031611. 